\title{
Frequent Premature Atrial Complex: A Neglected Marker of Adverse Cardiovascular Events
}

\author{
Raymond Pranata \\ Department of Cardiology and Vascular Medicine, Faculty of Medicine, Universitas Pelita Harapan, Tangerang, Banten, Indonesia \\ ORCID: \\ Raymond Pranata: https://orcid.org/0000-0003-3998-6551
}

\section{Abstract}

Premature atrial complexes (PACs) have long been considered a benign electrophysiological phenomenon unlikely to result in a severe clinical consequence. However, recent evidence had challenged this notion. Several studies showed that excessive PAC leads to the development of atrial fibrillation and subsequent hospitalization. The PAC is also associated with mortality, cardiovascular hospitalization, permanent pacemaker implantation, and nonlacunar ischemic stroke. The cutoff for excessive PAC differs from one study to another ranging from 76 PACs per $24 \mathrm{~h}$ to $>32$ PACs per hour. Increased adverse cardiovascular events demonstrated by recent studies had challenged the long-held paradigm that PACs are unlikely to result in serious clinical consequences.

Keywords: Atrial fibrillation, cardiovascular events, premature atrial complex, premature atrial contraction, stroke

\section{INTRODUCTION}

Premature activation of atria from a focus other than sinus node is known as supraventricular premature complexes also known as supraventricular extrasystoles. Majority of supraventricular premature beats arise from atria; however, it may also originate from the atrioventricular node (junctional premature beats). Premature atrial complexes (PACs) which are also referred as atrial premature beats, atrial premature complexes, premature atrial beats, premature atrial contraction, or atrial extrasystoles are triggered by the focus in the atrial myocardium. PAC has long been considered a benign electrophysiological phenomenon unlikely to result in serious clinical consequence. ${ }^{[1]}$ The information about the mechanisms of PACs in humans is not clear or well investigated since invasive testing is rarely performed in patients with only simple PACs. It seems to involve multiple mechanisms including re-entry within the atrium, abnormal automaticity, and triggered activity. The prevalence in young individuals has been reported to be $73 \%$ and $100 \%$ in apparently healthy older individuals. ${ }^{[2]}$ However, recent evidence had challenged this notion. Frequent PAC and subclinical atrial tachyarrhythmias

Submission: 21-03-2019 Revision: 26-05-2019 Accepted: 27-06-2019

Published Online: 13-02-2020

\begin{tabular}{|l|l|}
\hline \multicolumn{3}{|c|}{ Access this article online } \\
\hline Quick Response Code: & Website: \\
& http:/www.ijcva.com \\
& \\
\end{tabular}

have been shown to be strong and independent predictors of incident atrial fibrillation (AF) and associated complications. ${ }^{[3]}$ AF was associated with an increased risk of death, stroke, and congestive heart failure. ${ }^{[4]}$

\section{Risk Factors Associated with Development of Premature Atrial Complex}

Risk factors for PAC frequency included age, height, a history of cardiovascular disease, physical activity, natriuretic peptide levels, and low high-density lipoprotein (HDL) cholesterol but not hypertension and body mass index according to a study by Conen et al. ${ }^{[3]}$ The prevalence of PAC was described to be strongly increased with age (relative risk [RR] per standard deviation [SD]: $1.80 ; P<0.0001)$ from a median of $0.8 / \mathrm{h}$ among participants aged $50-55$ years to $2.6 / \mathrm{h}$ among those aged $\geq 70$ years which is threefold compared to the former. Height was associated with increased PAC frequency

Address for correspondence: Dr. Raymond Pranata, Department of Cardiology and Vascular Medicine, Faculty of Medicine, Universitas Pelita Harapan, Tangerang, Banten, Indonesia. E-mail: raymond_pranata@hotmail.com

This is an open access journal, and articles are distributed under the terms of the Creative Commons Attribution-NonCommercial-ShareAlike 4.0 License, which allows others to remix, tweak, and build upon the work non-commercially, as long as appropriate credit is given and the new creations are licensed under the identical terms.

For reprints contact: reprints@medknow.com

How to cite this article: Pranata R. Frequent premature atrial complex: A neglected marker of adverse cardiovascular events. Int J Cardiovasc Acad 2020;6:1-4. 
(RR per SD: $1.52 ; P<0.0001$ ), and the increased risk among taller individuals might be explained as a result of the close relationship between body size and left atrial (LA) size. A history of cardiovascular disease (RR: $2.40 ; P<0.0001)$ and log-transformed NT-proBNP (RR per SD: $1.27 ; P<0.0001$ ) were associated with increased PAC frequency as elevated levels of natriuretic peptides probably reflect a higher burden of subclinical and clinical structural heart disease and increased wall stress which in turn may favor PAC occurrence, while history of cardiovascular diseases showed clinical structural heart disease straightforwardly. ${ }^{[2,3]}$ HDL cholesterol (RR per SD: $0.80 ; P=0.0002$ ) and an inverse relationship with the frequency of PAC might be explained by the fact that cell membranes, which are a major determinant of cell excitability, might be affected by blood lipids. At least $2 \mathrm{~h}$ of physical exercise per day is associated with lower PAC counts (RR: $0.69 ; P=0.002$ ); however, a vigorous exercise was linked to increased risk of AF. Hypertension and obesity are associated with enlarged LA and are major risk factors for AF; however, it is surprising that hypertension and obesity are not found to be related to increased PAC counts. Height was associated with increased PAC frequency with enlarged LA as a possible explanation. Since the electrophysiological mechanism is not fully elucidated, this phenomenon cannot be explained theoretically. These findings are counterintuitive and require further investigations to confirm.

\section{Frequent Premature Atrial Complex as a Predictor of New-onset Atrial Fibrillation}

Frequent PACs were linked with new-onset AF; it is thought that atrial electrical activity plays a vital role in $\mathrm{AF}$ initiation and maintenance. In those with AF, PACs from the pulmonary veins frequently trigger AF. Chong et al. showed that frequent PACs (hazard ratio [HR]: 3.22; 95\% confidence interval [CI]: $1.9-5.5 ; P<0.001)$ to be an independent predictor for the new occurrence of AF. Chong et al. study enrolled those without AF or structural heart disease undergoing $24 \mathrm{~h}$ and electrocardiography and was followed up for 6.1. Chong et al. also found that age $>75$ years (HR: 2.3 ; 95\% CI: $1.3-3.9$; $P=0.004)$ and coronary artery disease (HR: $2.5 ; 95 \% \mathrm{CI}$ : $1.4-4.4 ; P=0.002$ ) were also found to be an independent predictor for the new occurrence of $\mathrm{AF}^{\left[{ }^{[2]}\right.}$ Binici et al. in cohort of the Copenhagen Holter Study, consisting of 678 healthy men and women aged between 55 and 75, showed that frequent PACs contribute to a nearly 3-fold increased risk of AF hospitalization (HR: 2.78; 95\% CI: 1.08-6.99;
$P=0.033$ ) in median follow-up of 6.3 years. Development of AF is more frequent in those with higher baseline systolic blood pressure (169.5 versus $155.9 \mathrm{~mm} \mathrm{Hg} ; P=0.02$ ) and older age (67.5 versus 64.4 years; $P=0.03) .{ }^{[5]}$ In adjusted analyses of Dewland et al. study, doubling hourly PAC counts were associated with a $17 \%$ increase in the development of AF over 10 years (HR: 1.17; 95\% CI: $1.13-1.22 ; P<0.001$ ). Specificity for predicting AF at 15 years exceeded $90 \%$ for PAC $>32$ beats/h. The participants were 1260 adults; the participants with incident AF were significantly older, were more likely to be male, and had a higher prevalence of hypertension, heart failure, and coronary artery disease. ${ }^{[6]}$ Lin et al. studied 5371 consecutive patients without AF or a permanent pacemaker (PPM) at baseline who underwent 24-h electrocardiography monitoring. They found that PAC burden $>76$ beats/day was independently associated with new-onset AF (HR: 1.757; 95\% CI: 1.427-2.163) ${ }^{[7]}$ [Table 1]. A meta-analysis by Patel et al. on six observational studies showed that frequent PACs were associated with new-onset AF (RR: $3.38 ; 95 \%$ CI: $1.63-7.01) .{ }^{[8]}$

To summarize, studies showed that frequent PACs were associated with an increased risk of developing new-onset AF. Other risk factors related to the development of AF in those patients were age, hypertension, heart failure, and coronary artery disease. These factors are also related to the development of PACs, while the aforementioned are also established risk for AF.

\section{Frequent Premature Atrial Complex as a Predictor of Stroke}

Chong et al. demonstrated that ischemic strokes were more frequent in patients with PACs (16 out of $41 ; 39 \%$ ) compared to those without $(25$ out of $41 ; 61 \%)$ with a HR of $2.1(95 \%$ CI: $1.1-4.8 ; P<0.001)$. Among these 41 ischemic stroke patients, eight developed new-onset AF prior to ischemic stroke. ${ }^{[2]}$ During the follow-up period in a study by Binici et al., 27 participants experienced a stroke. Ten events of stroke occurred in participants with excessive supraventricular ectopic activity (ESVEA)/frequent PAC and 17 in participants without (HR: 3.88; 95\% CI: $1.78-8.48 ; P=0.0007$ ). This finding remained significant after adjustment for age and sex (HR: 2.79; 95\% CI: 1.23-6.30; $P=0.014$ ) and remained significant after further adjustment for other major risk factors for stroke (smoking, diabetes mellitus, systolic blood pressure, and body mass index) (HR: 2.37; 95\% CI: 1.02-5.50;

\begin{tabular}{|c|c|c|c|c|c|}
\hline Study & Definition of Frequent PACs & Sample & Hazard ratio & 95\% confidence interval & $\boldsymbol{P}$ \\
\hline Chong, et al & top quartile of the present cohort (i.e. PACs 100 beats/day) & 428 & 3.22 & $1.9-5.5$ & $P<0.001$ \\
\hline Binici Z, et al & $\begin{array}{l}\geq 30 \text { supraventricular ectopic complexes (SVEC) per h or } \\
\text { as any episodes with runs of } \geq 20 \text { SVEC }\end{array}$ & 678 & 2.78 & $1.08-6.99$ & $P=0.033$ \\
\hline Dewland TA, et al & - & 1260 & 1.17 & $1.13-1.22$ & $P<0.001$ \\
\hline Lin $\mathrm{CY}$, et al & PAC $>76 /$ day & 5371 & 1.757 & $1.427-2.163$ & $P<0.001$ \\
\hline
\end{tabular}


$P=0.044) .{ }^{[5]}$ A study by O'Neal et al. showed that PACs were associated with an increased risk of ischemic stroke (HR: 1.34; 95\% CI: 1.04-1.74), although the relationship was limited only to nonlacunar infarcts (HR: 1.42; 95\% CI: 1.08-1.87), and not lacunar strokes (HR: 1.01; 95\% CI: 0.51-2.03). Association between PACs and ischemic stroke was stronger among women (HR: 1.82; 95\% CI: 1.29-2.56) than men (HR: 1.03; 95\% CI: $0.69-1.52 ; P=0.0095)^{[9]}$ [Table 2]. A meta-analysis by Patel et al. on six observational studies showed that frequent PACs were associated with risk of stroke (RR: 2.88 ; 95\% CI: 1.71-4.84.)

\section{Frequent Premature Atrial Complex as a Predictor of Congestive Heart Failure, Mortality, and Cardiovascular Hospitalization}

Frequent PACs are associated with congestive heart failure and mortality. Chong et al. study stated that in those with frequent PACs who are more likely to develop congestive heart failure (HR: 2.2; 95\% CI: $1.2-5.6 ; P=0.02$ ) and in those (35 patients) who developed congestive heart failure, only two were preceded the development of AF. They are also related with higher mortality (HR: 1.8; 95\% CI: $1.1-3.6, P=0.02) .{ }^{[2]}$ Binici et al. study founded that 21 of the participants with ESVEA (21/99) and 66 of the participants without ESVEA (66/579) died during the follow-up (HR: $2.12 ; 95 \% \mathrm{CI}: 1.30-3.47 ; P=0.003$ in univariate analysis). However, after adjustment for conventional risk factors, ESVEA became statistically nonsignificant (HR: $1.40 ; 95 \%$ CI: $0.83-2.37 ; P=0.207) .{ }^{[5]}$ In adjusted analyses of Dewland et al. study, doubling hourly PAC counts were associated with $6 \%$ increase in overall mortality (HR: $1.06 ; 95 \%$ CI: $1.03-1.09 ; P<0.001){ }^{[6]}$ Lin et al. showed that the PAC burden $>76$ beats/day was independently associated with mortality (HR: 1.384; 95\% CI: 1.230-1.558) and cardiovascular hospitalization (HR: 1.284; 95\% CI: 1.137-1.451) ${ }^{[7]}$ [Table 3]. A meta-analysis by Patel et al. on six observational studies showed that frequent PACs were associated with higher all-cause mortality (RR: 2.23; 95\% CI: 1.93-2.59) and cardiovascular mortality (RR: 3.07; 95\% CI: 2.63-3.59). ${ }^{[8]}$

\section{Frequent Premature Atrial Complex and Other Cardiac Conductive Dysfunctions}

Last but not the least, it is also associated with higher rate of PPM implantation (HR: 2.821; 95\% CI: 1.898-4.192) in the long term as reported by Lin et al. Mechanism for the association between PACs and cardiac conduction system dysfunction was unclear. Frequent atrial stimulation might cause atrial substrate remodeling near the sinus nodal area, causing dysfunction of the sinus node. ${ }^{[7]}$

To summarize, frequent PACs may not as benign as it seemed to be. Recent evidence showed that frequent PACs are associated with adverse cardio-cerebrovascular events in the long term. Adverse events include new-onset AF, ischemic

\begin{tabular}{|c|c|c|c|}
\hline Study & $\begin{array}{l}\text { Hazard } \\
\text { ratio }\end{array}$ & $\begin{array}{l}\text { 95\% confidence } \\
\text { interval }\end{array}$ & $P$ \\
\hline Chong, et al & 2.1 & $1.1-4.8$ & $P<0.001$ \\
\hline Binici Z, et al & 2.37 & $1.02-5.50$ & $P=0.044$ \\
\hline O'Neal WT, et al & 1.34 & $1.04-1.74$ & $P<0.001$ \\
\hline
\end{tabular}

\begin{tabular}{lccc} 
Table 3: Frequent PACs are related to increased mortality \\
\hline Study & $\begin{array}{c}\text { Hazard } \\
\text { ratio }\end{array}$ & $\begin{array}{c}\text { 95\% confidence } \\
\text { interval }\end{array}$ & $\boldsymbol{P}$ \\
\hline Chong, et al & 1.8 & $1.1-3.6$ & $P=0.02$ \\
Binici Z, et al & 1.4 & $0.83-2.37$ & $P=0.207$ \\
Dewland TA, et al & 1.06 & $1.03-1.09$ & $P=0.025$ \\
Lin CY, et al & 1.384 & $1.230-1.558$ & $P<0.001$ \\
\hline
\end{tabular}

stroke (especially nonlacunar infarction), congestive heart failure, higher overall mortality, and PPM implantation. Now, there is no definite cutoff for treatment for patients with excessive PACs, and the cutoff for excessive PAC differs from one study to another ranging from 76 PACs per $24 \mathrm{~h}$ to $>32$ PACs per hour. Patel et al. in their meta-analysis in 2017 stated that studies generally defined frequent PACs as occurring $\geq 1$ time during a standard ECG recording or $\geq 30$ times over a 1-h recording or any episodes with runs of $\geq 20$ PACs. Lifestyle changes by reducing the intake of triggers (if identified) such as caffeine, tobacco, alcohol, or other substances should be encouraged in patients with excessive PACs. However, there is insufficient evidence for pharmacological treatment in excessive PACs. Currently, PAC count would be valuable as a prognostication tool rather than a threshold for treatment. Further trials on PAC suppression should be done to clarify the role of pharmacological therapy.

\section{Conclusion}

Increased adverse cardiovascular events and mortalities demonstrated by recent trials had challenged the long-held paradigm that PACs are unlikely to result in serious clinical consequences. It is also suggested by some authors that frequent PACs may be used as a prediction tool in predicting new-onset AF. The author believes that frequent PACs may be a neglected marker of cardiovascular catastrophe and deserve more attention at least on the same level as other conventional risk factors.

\section{Financial support and sponsorship}

Nil.

\section{Conflicts of interest}

There are no conflicts of interest.

\section{References}

1. Marcus GM, Dewland TA. Premature atrial contractions: A wolf in sheep's clothing? J Am Coll Cardiol 2015;66:242-4. 
2. Chong BH, Pong V, Lam KF, Liu S, Zuo ML, Lau YF, et al. Frequent premature atrial complexes predict new occurrence of atrial fibrillation and adverse cardiovascular events. Europace 2012;14:942-7.

3. Conen D, Adam M, Roche F, Barthelemy JC, Felber Dietrich D, Imboden $\mathrm{M}$, et al. Premature atrial contractions in the general population: Frequency and risk factors. Circulation 2012;126:2302-8.

4. Conen D, Chae CU, Glynn RJ, Tedrow UB, Everett BM, Buring JE, et al. Risk of death and cardiovascular events in initially healthy women with new-onset atrial fibrillation. JAMA 2011;305:2080-7.

5. Binici Z, Intzilakis T, Nielsen OW, Køber L, Sajadieh A. Excessive supraventricular ectopic activity and increased risk of atrial fibrillation and stroke. Circulation 2010;121:1904-11.

6. Dewland TA, Vittinghoff E, Mandyam MC, Heckbert SR, Siscovick DS,
Stein PK, et al. Atrial ectopy as a predictor of incident atrial fibrillation: A cohort study. Ann Intern Med 2013;159:721-8.

7. Lin CY, Lin YJ, Chen YY, Chang SL, Lo LW, Chao TF, et al. Prognostic significance of premature atrial complexes burden in prediction of long-term outcome. J Am Heart Assoc 2015;4:e02192.

8. Patel A, Anugula D, Kanmanthareddy A, Urja P, Haddad TM, Alla V. Prognostic impact of premature atrial complexes in the general population: A meta-analysis of observational studies. J Am Coll Cardiol 2017;69 11 Suppl: 333.

9. O'Neal WT, Kamel H, Kleindorfer D, Judd SE, Howard G, Howard VJ, et al. Premature atrial contractions on the screening electrocardiogram and risk of ischemic stroke: The reasons for geographic and racial differences in stroke study. Neuroepidemiology 2016;47:53-8. 